\title{
Reflexiones acerca de los aspectos epistemológicos de la agroecología
}

Lizeth Marelly Álvarez-Salas*

Diana Nayibe Polanco-Echeverry ${ }^{* *}$

Leonardo Ríos-Osorio ${ }^{* * *}$

Recibido: 20I4-OI-28 Aprobado: 20I4-05-I4 Disponible en línea: 20I4-07-27

doi:Io.III44/javeriana.CRDII-74.raea

Cómo citar este artículo: Álvarez-Salas, L., Polanco-Echeverry, D., Ríos-Osorio, L. (20I4). Reflexiones acerca

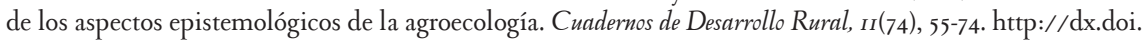
org/I0.III44/Javeriana.CRDII-74.raea

\section{Resumen}

Este artículo de reflexión teórica busca continuar la discusión sobre un enfoque epistemológico en el cual se circunscribe a la agroecología como una ciencia de la complejidad, transdisciplinar y de carácter sistémico, orientada a dar respuesta a los problemas en los agroecosistemas. Para ello, se propone como objeto de estudio la resiliencia socioecológica y como objeto de conocimiento los agroecosistemas. Se espera que la definición de estos límites de la acción investigativa permita la consolidación de la agroecología en el campo del conocimiento.

\section{Palabras clave:}

agroecología; epistemología; transdisciplinariedad; pensamiento complejo

\footnotetext{
* Bióloga, Magister en Antropología y PhD (c) en Agroecología de la Universidad de Antioquia. Docente e investigadora de la Universidad de Antioquia. Correo electrónico: lizethalvarezsalas@gmail.com

** Bacterióloga y laboratorista Clínica (Institución Universitaria Colegio Mayor de Antioquia, Colombia). Magíster en Ciencias Biológicas (Universidad del Tolima, Colombia). PhD(c) en Agroecología (Universidad de Antioquia, Colombia). Investigadora y docente de la Universidad de Antioquia.

Correo electrónico: dianapolancoecheverry@gmail.com

${ }^{* * *}$ Bacteriólogo (Universidad de Antioquia). Especialista en Parasitología Humana (Universidad de Antioquia); PhD en Sostenibilidad, Tecnología y Humanismo (Universitat Politécnica De Catalunya). Docente e investigador de la Universidad de Antioquia. Correo electrónico: lrios_oI@hotmail.com
}

\section{c) $0 \$ \theta$}




\title{
Reflections on the Agroecology Epistemological Aspects
}

\begin{abstract}
This reflective theoretical article seeks to continue the discussion on an epistemological approach in which agroecology is circumscribed as a science of complexity, transdisciplinary and systemic in nature, aimed at responding to the problems of agroecosystems.To do this, the socio-ecological resilience is proposed as an object of study and the agroecosystems as a knowledge object. It is expected that the definition of these limits of action research enables the consolidation of agroecology in the field of knowledge.
\end{abstract}

\section{Keywords:}

agroecology; epistemology; transdisciplinarity; complex thinking

\section{Réflexions sur les aspects épistémologiques de l’agroécologie}

\section{Résumé}

Cet article de réflexion théorique vise à poursuivre la discussion sur une approche épistémologique qui circonscrit l'agroécologie comme une science de la complexité, transdisciplinaire, et de caractère systémique, qui s'intéresse à résoudre les problèmes des agro-écosystèmes. Pour cela, la résilience socio-économique est proposée comme un objet d'étude et les agro-écosystèmes comme des objets de connaissance. On attend que la définition de ces limites de la recherche-action permette la consolidation de l'agroécologie dans le domaine du savoir.

\section{Mots-clés:}

agroécologie ; épistémologie; transdisciplinaire; pensée complexe 


\section{Introducción}

En el marco actual de las ciencias, existe la necesidad de desarrollar nuevos enfoques de investigación que se aproximen a la compleja realidad que se percibe en el mundo; la ciencia como sistema de pensamiento ha evolucionado de la mano de las evidencias cada vez más acuciantes de un mundo en crisis. Fenómenos como el calentamiento global, crisis económicas que ponen en peligro la estabilidad de países enteros y por ende a sus poblaciones. La creciente problemática de pobreza y la hambruna mundial son el detonante de la crisis del paradigma cartesiano de la ciencia clásica, que evidencia su falta de efectividad para dar respuesta a estas situaciones prioritarias en las agendas científicas.

En este sentido, en su debate sobre las dos culturas, Snow (2000) planteaba cómo la separación entre ciencias duras y blandas, en el contexto de las ciencias clásicas, había disgregado el abordaje de la complejidad de la realidad del mundo en materia y forma. Además, alertaba sobre cómo esta situación había llevado a los científicos a romper los canales de comunicación entre estas formas de hacer investigación.

A raíz de las reflexiones, han emergido los enfoques de la complejidad para dar respuesta a la crisis de insostenibilidad que afecta a la civilización. Al superar los atavismos de los modelos clásicos de la investigación científica, las ciencias nuevas no son la simple fusión de enfoques cualitativos y cuantitativos, son una proyección de la complejidad del mundo sobre la complejidad en el abordaje de esa realidad.

La agroecología, un ejemplo de estas ciencias nuevas, se ha desarrollado como una alternativa para comprender los sistemas agroecológicos. Sin embargo, es evidente que no existe aún claridad alrededor de sus postulados científicos y que se ha denominado agroecología a investigaciones que no se corresponden con los principios propuestos por ella (Gómez, Ríos-Osorio y Eschenhagen, 2013). Por lo anterior, se hace necesario desarrollar un cuerpo teórico alrededor de los aspectos epistemológicos de esta ciencia, que permitan establecer lineamientos tanto teóricos como metodológicos para la construcción de su objeto de estudio y de sus propias preguntas de investigación.

Este artículo de reflexión pretende hacer un aporte a la discusión sobre los aspectos epistemológicos de la agroecología, con una propuesta de objetos de estudio, de conocimiento y unos enfoques epistemológicos aplicables a la aprehensión de su objeto. Este es un aporte a la consolidación disciplinar y a la proyección de la agroecología como un espacio de formación científica. 


\section{Antecedentes de la epistemología agroecológica}

El término "agroecología” aparece por primera vez a principios del siglo XX (Wezel, Bellon, Doré, Francis, Vallod y Davis, 2009, p. 2), articulado con la evolución de la agronomía, la ecología, la zoología, la botánica, la fisiología de las plantas y sus aplicaciones en temas agrícolas y medioambientales. Wezel et al., distinguen dos momentos históricos: el primero entre 1930 y 1960, cuando se obtuvieron logros en la investigación sobre el control de plagas, el manejo de suelos, la predicción de variables climáticas y su impacto en la agricultura y la economía. Un segundo momento se define entre 1960 y 2000, años que marcan el inicio y desarrollo de la revolución verde, cuyo propósito central fue aumentar la producción de alimentos para diezmar el hambre en países en vía de desarrollo. Sin embargo, este modelo ha aumentado las áreas productivas a costa de la disminución de la diversidad, ha incrementado la fragilidad de los ecosistemas, la contaminación de los suelos, del agua y del aire y de semillas criollas por transgénesis (Hoobbelink, 1987).

Estos efectos de la agricultura moderna justifican que entre los años 1960 y 2000 la agroecología se constituyera como una alternativa a este modelo de desarrollo rural para encarar la crisis ecológica y los problemas en el agro, a partir del manejo sostenible de los recursos naturales y del acceso igualitario a estos (Wezel et al., 2009). En este proceso surgen tres visiones que orientan la consolidación de la agroecología desde los años sesenta hasta la actualidad. La primera visión la considera como una práctica emergente e innovadora desde el punto de vista tecnológico, relacionada con un proceso de consolidación sociocultural a partir de la comprensión de dinámicas ecológicas aplicadas a los cultivos y al manejo de los recursos naturales en América Latina y el Caribe (Toledo, 20I2).

Esta visión se fortaleció en la década de los años noventa, posterior al surgimiento de los movimientos ecologistas surgidos en la década de 1960 en Estados Unidos, que objetaron tanto la agricultura industrial como el deterioro ecológico y ambiental. La segunda visión refiere a la agroecología como un movimiento social, cultural y político (Vía Campesina y el Movimiento Sin Tierra [MST]) orientado al empoderamiento del campesinado, a la distribución equitativa de la tierra y a la reformulación de dinámicas económicas incluyentes y justas (Holt y Patel, 2013). Una tercera visión la cataloga como una disciplina científica en consolidación epistemológica y metodológica que, de acuerdo con Gómez (et al., 2013), ha estado marcada por un fenómeno de "colonialidad" del saber. Las preguntas son formuladas principalmente en centros de investigación de países del "primer mundo” y resueltas en países en "vía de desarrollo". A esta visión se hará referencia en este artículo. 
En los años ochenta, la agroecología se propuso como una disciplina que usaba métodos holísticos para el estudio de los agroecosistemas, con el fin de proteger recursos naturales mediante pautas para su diseño y gestión (Gliessman, 2002). En este sentido, Conway (1987) identificó cuatro propiedades principales de los agroecosistemas: productividad, estabilidad, sostenibilidad y equidad. Esta influencia contribuyó a la construcción del concepto de sostenibilidad para la agricultura (Altieri, 1995). En esta época, se hizo referencia al concepto de agroecosistema desarrollado por el ecólogo Odum (1969, en Altieri, 1995), quien los considera como "ecosistemas domesticados", una interfaz entre los ecosistemas naturales y los fabricados.

Posteriormente, Altieri (1999, p. 9) redefine la agroecología como "una disciplina que provee los principios ecológicos básicos para estudiar, diseñar y manejar agroecosistemas que sean productivos y conservadores del recurso natural, y que también sean culturalmente sensibles, socialmente justos y económicamente viables”. Norgaard (1997) afirma que la investigación agroecológica se interesa no solo por la maximización de la producción, sino por la optimización del agroecosistema total.

Los principios agroecológicos que refiere Altieri fueron postulados previamente por Reijntjes, Haverkort y Waters-Bayer (I992, pp. 6I-98) como: a) diversificación vegetal y animal en cuanto a especies o genética en tiempo y en espacio; b) reciclaje de nutrientes y materia orgánica, optimización de la disponibilidad y balance del flujo de nutrientes; c) provisión de las condiciones edáficas necesarias para el crecimiento de cultivos, optimización del uso de materia orgánica y estímulo a la biología del suelo; d) minimización de pérdidas de suelo y agua, conservación de la cobertura, control de la erosión y manejo del microclima; e) minimización de pérdidas por insectos, microorganismos patógenos y malezas, con estímulo a la fauna benéfica, antagonistas y la alelopatía; y f) aprovechamiento de sinergias plantasmicroorganismos, planta-planta, plantas-animales y animales-animales. Este enfoque permite el rediseño de agroecosistemas más sostenibles basados en el conocimiento de los factores ecológicos aplicados a la finca.

Hasta este momento, el discurso agroecológico se desarrolló en un ámbito agronómico y ecológico, el cual se constituyó en el marco teórico de la investigación en esta disciplina. Hecht (1999, p. 17) explica cómo con frecuencia la agroecología conjuga ideas sobre "un enfoque de la agricultura más ligado al medio ambiente y más sensible socialmente; centrada no solo en la producción sino también en la sostenibilidad ecológica del sistema de producción”. Expresión que ella cataloga 
como, “uso 'normativo o prescriptivo' del término agroecología”, y sostiene que la agroecología aborda tanto el estudio de fenómenos netamente ecológicos dentro del campo de cultivo, como las relaciones ecológicas entre los organismos del sistema. Por ende, la investigación agroecológica se concentra en asuntos puntuales del área de la agricultura, pero dentro de un contexto más amplio que incluye variables ecológicas y sociales. Sin embargo, la autora no profundiza en las estrategias metodológicas que establezcan las particularidades socioculturales que humanizan los agroecosistemas, sino que se limita al análisis de sus variables biofísicas.

Para Hecht la agroecología es un enfoque que integra ideas y métodos de varios subcampos; es más que una disciplina específica y puede ser un desafío normativo de las disciplinas que abordan las preguntas y problemas en el ámbito agrícola. Explica, además, que este enfoque tiene sus raíces en las ciencias agrícolas, en el movimiento del medio ambiente, en la ecología, en el análisis de agroecosistemas indígenas y en los estudios sobre el desarrollo rural. Concluye que, a pesar de que son áreas de investigación con objetivos y metodologías diferentes, han influenciado de manera importante el pensamiento agroecológico.

Una aproximación al análisis de componentes socioculturales en agroecología, fue la introducción de métodos etnobotánicos y etnoecológicos que buscaban comprender y replicar los conocimientos indígenas y campesinos aplicados a la producción de alimentos en sistemas productivos tradicionales (Barrera-Bassols y Toledo, 2005). Por esta razón, este bagaje cognitivo se constituyó como un principio agroecológico más, aunque no sea explícito o suficientemente abordado.

A este respecto, es necesario establecer una distinción entre los conocimientos tradicionales y locales, ya que se usan en el área de forma indistinta. Los conocimientos tradicionales se desarrollan en grupos étnicos amerindios, asiáticos y africanos, e incluyen valores culturales y creencias míticas que surgen a partir de la cosmovisión de esas sociedades y son trasmitidos en forma oral. Algunos son exclusivos de individuos con una importancia religiosa o política (Zerda, 2003). De otro lado, los conocimientos locales surgen de manera informal desde la observación de los agroecosistemas, y son una mezcla de sentido común, interacciones sociales y prácticas. Además, se adaptan a cambios introducidos a partir de la ciencia formal, son dinámicos, acumulativos y no son exclusivos de los grupos étnicos (MoraDelgado, 2008).

Al respecto, además de establecer la importancia de comprender factores socioculturales que rodean la práctica productiva en los análisis agroecológicos, León (2012) sugiere la agroecología como una instancia para rescatar los 
conocimientos tradicionales. Sin embargo, más allá de esta pretensión, es menester comprender la racionalidad, los procesos de trasformación y la vigencia de estos conocimientos. En ese aspecto, Ulloa (200I) plantea la improcedencia de establecer estrategias de rescate de conocimientos tradicionales por parte de la academia, ya que parte de una noción esencialista de las comunidades. Su pérdida o trasformación obedecen a fenómenos culturales tanto endógenos como exógenos propios del proceso de globalización. Como se explicó, los conocimientos tradicionales están sujetos a la cosmovisión particular y funcional en la cultura; por tanto, su aplicación en unidades productivas externas estaría restringida al ámbito biofísico como una mera técnica aplicada a la producción.

Si bien el intento de comprender los conocimientos tradicionales o locales es un aporte valioso para el desarrollo de una perspectiva agroecológica más compleja, esto no resuelve la pretensión de incluir determinantes socioculturales en el análisis de los agroecosistemas. De forma paralela al desarrollo de una perspectiva agroecológica con enfoque ecológico-productivo, Sevilla (20II, pp. I09-I25) plantea la agroecología como una alternativa para el desarrollo rural sostenible en América Latina. Este fenómeno se fortalece desde el rol sociopolítico del campesino en la agroecología, desarrollado por Holt y Patel (20I0). Sin embargo, estas variables sociales no se incluyen en los principios agroecológicos que definen los agroecosistemas, por tanto, son analizados desde métodos sociales que ofrecen una óptica fragmentada de la realidad.

Caporal, Costabeber y Paulus (2009) consideran que la agroecología debe contribuir al análisis prospectivo multidimensional: económico, social, ambiental, cultural, político y ético. Debe tener también un enfoque metodológico y teórico propio, que se nutra con el aporte de diversas disciplinas científicas, para contribuir a la emergencia de un nuevo paradigma de desarrollo rural sostenible. En este sentido, la diversidad sociocultural, ecológica y la participación activa de las comunidades en los procesos de investigación, caracterizan el enfoque agroecológico.

Esta noción es retomada por León y Altieri (2010, en León 20I2, p. 32), quienes proponen definir la agroecología como "la ciencia que estudia la estructura y función de los agroecosistemas tanto desde el punto de vista de sus interrelaciones ecológicas como culturales”. De este modo se evita la especialización del conocimiento y se interesa más en las interrelaciones de factores biofísicos, ecosistémicos y culturales. Además, abordan el análisis de su objeto de estudio desde la complejidad que implican estas interrelaciones, da valor al conocimiento de campesinos, indígenas y afrodescendientes, y toma en cuenta todos los efectos ambientales que son consecuencia del uso de conocimientos especializados. 
Esta reflexión de los agroecólogos supera, en apariencia, la noción técnicoagrícola-ecológica, al introducir en la investigación el componente sociocultural. Supone el abordaje en profundidad desde Morin (1999) y Caporal et al. (2009), al integrar el pensamiento complejo al análisis de sistemas, y define la agroecología como una ciencia para la sostenibilidad que permite una mirada más compleja y holística de los sistemas de producción y sus fenómenos. Además, menciona que puede ser vista como una ciencia enmarcada en el paradigma de la complejidad, derivado a su vez de una especificidad de la teoría general de sistemas propuesta por Ludwig Von Bertalanffy en 1989. Precisamente, de esta teoría se deriva la presunción de algunos autores que hacen referencia al pensamiento complejo como aquel que "busca, al mismo tiempo, distinguir - pero sin desunir - y religar" (Morin, I999; Caporal et al., 2009, p. 67).

Lo anterior soporta la idea de que la agroecología se acerca a su objeto de estudio desde los sistemas complejos (Gallopin, 200I), e incluye todos los componentes del agroecosistema sin descuidar su multidimensionalidad. De otro lado, Caporal et al. (2009) menciona que la forma como las partes se relacionan hace emerger nuevas propiedades. Esta perspectiva la separa del paradigma cartesiano que simplifica, reduce y fracciona al objeto de estudio. Este enfoque emplaza a la agroecología en el campo de los sistemas complejos, y la constituye en una ciencia que aborda su objeto de estudio desde un enfoque epistemológico multidimensional. Para Toledo (20I2) la confluencia de todas estas áreas de conocimiento lleva a la agroecología a constituirse como una disciplina híbrida, que resulta de las reacciones al proceso de parcelación y especialización excesiva de la ciencia contemporánea.

La revisión de algunas publicaciones en agroecología demuestra que la investigación en esta área es aún dispersa y no responde a una visión unificada del objeto disciplinario de estudio. Por ejemplo, desde la perspectiva cuantitativa la agroecología ha tenido significativos avances en el entendimiento del uso de la biodiversidad y la agrodiversidad como un factor para el incremento de la producción (Altieri et al., 20I2), así como también de los servicios ecosistémicos que aportan a los sistemas productivos (Abbona, 2007), la eficacia energética de los mismos (Martínez-Negrete, Martínez, Joaquín, Sheinbaum y Masera, 2013), entre otros. Mientras que desde la cualitativa, se han realizado estudios sobre las actitudes de los campesinos en relación con la conservación de la biodiversidad (Junge, Jacot, Bosshard y Lindemann-Matthies, 2009), análisis teóricos sobre los movimientos sociales rurales (Rosset y Martínez-Torres, 20I2) y otros corte fenomenológico. 
Es posible que esta multiplicidad de enfoques en un área en consolidación sea producto de un entrenamiento formal disciplinar en lugar de uno sistémico y transdisciplinario de los investigadores (Gómez et al., 20I3). Varias preguntas surgen a raíz de esta reflexión, por ejemplo: ¿cuál es el tipo de investigación que debe hacer un profesional con formación científica en agroecología? ¿Cómo se garantiza que las preguntas de investigación respondan al contexto del cual surgen? Está claro que no cualquier estudio, bien desde la perspectiva ecológica de las plantas o el suelo aplicado a la agricultura, o un análisis sociopolítico de los movimientos campesinos, es un estudio agroecológico. El enfoque que se plantea en este artículo invita a poner en común elementos mínimos relacionados con los aspectos epistemológicos de la agroecología, su alcance y sus límites con respecto a otras disciplinas afines.

\section{El objeto de estudio y el objeto de conocimiento de la agroecología}

La evolución de la agroecología supone algunos acercamientos a la definición de su objeto de estudio; sin embargo, este es aún un tema en discusión. De acuerdo con Vargas (2003), la dificultad para llegar a un consenso sobre la definición del objeto de estudio retrasa la construcción de teorías y conceptos. En el caso de la agroecología ha supuesto una fragmentación del objeto de estudio, determinada por el enfoque disciplinar de cada investigador. Esto se evidencia en la mayoría de las investigaciones, que están orientadas a resolver problemas referidos a los componentes ecológicos-productivos o sociales, y no desde el análisis de los procesos y las relaciones socioecológicas que se establecen en el agroecosistema.

A este respecto, Salas-Zapata Ríos-Osorio y Álvarez-Del Castillo (20II, p. 702) formulan una interesante propuesta, donde "la resiliencia socioecológica de los agroecosistemas" se constituye como el objeto de estudio de la agroecología. Es importante aclarar que si bien el término "resiliencia" se construye a partir de la teoría ecológica (Holling, 1973), en la agroecología se entiende como la capacidad de adaptación a fenómenos ecológicos, sociales, culturales y ambientales que perturban el sistema de producción. Es, por tanto, la capacidad de un sistema para absorber las perturbaciones y reorganizarse de acuerdo con sus nuevas particularidades, con el fin de conservar, en esencia, la misma función, estructura e identidad (Walker, Holling, Carpenter y Kinzig 2004, p. 5). A su vez, la adaptabilidad en un sistema complejo es la capacidad de los actores para influir en su resiliencia. 
El estudio de la resiliencia socioecológica de los agroecosistemas responde mejor a las problemáticas analizadas y los fenómenos abordados por la agroecología, los cuales son temporales y dinámicos. La definición de este objeto de estudio exige establecer también un objeto de conocimiento. Es así como, desde la mirada agroecológica, el concepto de "sistema productivo" no es apropiado, pues surgió junto con la revolución verde. Los sistemas productivos así entendidos son un conjunto de componentes de un sistema mayor, que pueden ser estudiados de manera aislada; nada más alejado de la mirada sistémica donde un sistema no es un conjunto de partes. Aquí surge el cuestionamiento del por qué se sigue pensando que este concepto responde a un agroecosistema, aun cuando se sabe que no significa lo mismo desde la dimensión agroecológica.

Por tanto, el objeto de conocimiento de la agroecología es el agroecosistema, que es al mismo tiempo un lugar físico y un sistema naturaleza-sociedad, en que existe una reciprocidad entre componentes subjetivos y objetivos (Malpartida y Lavanderos, 1995). El agroecosistema es a su vez el objeto de otras disciplinas: es estudiado por el agrónomo desde la lectura biofísica, el zootecnista desde lo administrativo, el biólogo desde lo ecológico, y el sociólogo y antropólogo estudian sus características socioculturales; incluso lo trasciende hasta el concepto de sistemas agroalimentarios (Méndez y Gliessman, 2002).

Para la agroecología, la diferencia fundamental radica en que el agroecosistema trasciende los límites biofísicos de la parcela o el predio agrícola. Méndez y Gliessman (2002) explican cómo la agroecología incorpora en su objeto de conocimiento elementos como las políticas públicas, que si bien no son parte del agroecosistema, lo afectan y lo dinamizan. De esta manera, el agroecosistema se considera como la dimensión espacial donde se establecen las relaciones dinámicas entre los componentes del sistema. En este, las relaciones fluctúan y se trasforman de acuerdo con los proceso adaptativos; es decir, configuran una historia particular y única. Estos agroecosistemas son estructural y funcionalmente complejos, debido a las interacciones que se establecen entre los procesos ecológicos y socioculturales. Las interacciones pueden hacer emerger cualidades nuevas que solo son explicadas desde las relaciones entre los componentes.

Arnold y Osorio (1998) sugieren que para estudiar estos sistemas se deben considerar algunas de sus características básicas, establecidas por la teoría general de sistemas. Estas son: a) tienen atributos y propiedades estructurales y funcionales que caracterizan los componentes de un sistema; b) son circulares, esto hace referencia a procesos de autocausación y no a los de causa efecto; c) son complejos, lo que indica 
la cantidad de elementos del sistema, sus potenciales interacciones y la variabilidad de estas interacciones; d) en estos la energía se incorpora a los sistemas y se comporta según la primera ley de la termodinámica; e) tienden a la progresiva desorganización que los lleva a la homogenización con su ambiente; f) en sistemas abiertos el equilibrio sistémico solo es posible por la entrada de insumos del ambiente, que pueden ser flujos energéticos, de materiales o de información; g) tienen elementos emergentes que surgen a partir de las interacciones entre los componentes; $\mathrm{h}$ ) son homeostáticos, lo que hace referencia a los procesos internos que pretenden mantener invariable la estructura sistémica, con el fin de conservar su forma ante variaciones en el ambiente; i) y reciben información del ambiente. El análisis de estas características permitiría comprender los mecanismos de la resiliencia socioecológica de los agroecosistemas.

\section{Un enfoque epistemológico en agroecología}

La investigación agroecológica debe estar enmarcada en un enfoque epistemológico que no solo permita la comprensión y el estudio de la resiliencia socioecológica de los agroecosistemas, sino que además aborde los fenómenos desde la complejidad y la multidimensionalidad; esto, sobre la base de que son sistemas no lineales, presentan emergencias y procesos centrales. Para ello se debe tener en cuenta que los actores más relevantes son las personas con injerencia sobre el sistema de producción, en todos sus niveles. De estos actores conviene indagar y analizar sus características sociales y culturales y el conocimiento que tiene del agroecosistema.

Con miras a fortalecer la visión holística en la práctica investigativa, se plantea que el enfoque epistemológico más adecuado para la agroecología tiene como base la teoría de los sistemas complejos (Gallopin, 200I; García, 2006). Para su estudio, se deben comprender las interacciones que se establecen entre sus componentes, con el fin de explicar los fenómenos que se presentan dentro del sistema. Lo anterior implica ubicarse en un nivel transdisciplinario, que permita entender la naturaleza material del objeto de estudio, los tipos y las complejidades de los fenómenos del agroecosistema. Este enfoque es también complementario con la cibernética de segundo orden y la epistemología política (Ríos y Mesa, 2009), por tanto, un investigador puede acercarse a la pregunta de investigación desde uno o varios de ellos. A continuación se profundizará en el enfoque sistémico. 


\section{Enfoque sistémico}

En la obra de Morin (2003, p. 42), se describe un sistema "como una unidad compleja, un ‘todo' que no se reduce a la 'suma' de sus partes constitutivas”. Este sistema es, a su vez, abierto al disponer de una fuente energética externa, concepto aplicable a los organismos vivos y a los sistemas vivientes, que requieren de flujos de materiales y energía y flujos organizacionales e informacionales. Lo anterior permite comparar o relacionar los sistemas abiertos con los ecosistemas que los contienen. De acuerdo con el autor (pp. 44-45), y en referencia a la complejidad que caracteriza los sistemas abiertos:

“La realidad está, de allí en más, tanto en el vínculo como en la distinción entre el sistema abierto y su ambiente. Ese vínculo es absolutamente crucial desde el punto de vista epistemológico, metodológico, teórico, empírico. Lógicamente, el sistema no puede ser comprendido más que incluyendo en sí al ambiente, que le es a la vez íntimo y extraño y es parte de sí mismo siendo, al mismo tiempo, exterior”.

Adicional a la complejidad, otra característica atribuible a tales sistemas es la multidimensionalidad; en lo que concierne a los agroecosistemas, esta es fundamental para su análisis, como en el caso de la sociedad que "comporta dimensiones históricas, económicas, sociológicas y religiosas” (Morin, 2003, p. 15).

La multidimensionalidad de los agroecosistemas condiciona las metodologías para su estudio, a partir de las interacciones entre estos y su matriz ecosistémica. Por lo anterior se puede considera, que el sistema se desborda y trasciende a un nivel jerárquico superior de organización, o metasistema, y lo orienta sin duda a la complejidad. Morin (1999) afirma que el conocimiento podrá ser aplicable a la solución de problemas de la realidad mientras sea capaz de abordar la complejidad inherente a tales problemas, cuando se reconocen y comprenden los elementos constitutivos del sistema o de un todo como elementos indisolubles. La complejidad así entendida es, de acuerdo con Morin (1999, p. 16), "la unión entre la unidad y la multiplicidad”. En consecuencia, la investigación debe propender por el entendimiento de lo complejo, de su contexto, de una forma multidimensional en correspondencia con lo global.

García hace una aproximación teórico-metodológica en la manera como se abordan problemas desde la complejidad, que pueden ser aplicados a investigaciones agroecológicas. En su opinión, Morin no consolidó en sus aproximaciones una "formulación precisa de los problemas que enuncia (problemas que el cartesianismo 
dejó pendientes y que corresponden al campo de la teoría del conocimiento) como para conducir a una metodología de trabajo” (2006, p. 2I); sin embargo, esta fue la base teórica para los planteamientos posteriores.

El autor precisa que un sistema complejo es "una representación de un recorte de la realidad compleja, conceptualizado como una totalidad organizada (de ahí la denominación de sistema) en la cual los elementos no son 'separables' y por lo tanto no pueden ser estudiados aisladamente” (García, 2006, p. 2I). Además, completa esta definición al deducir que "investigar [estos] sistemas significa estudiar un 'trozo de la realidad' que incluye aspectos físicos, biológicos, sociales, económicos y políticos” (2006, p. 47).

La agroecología se plantea la necesidad de indagar sobre las interacciones socioecológicas que ayudan a entender los diversos fenómenos que se presentan en el agroecosistema. Debido a esto, un primer ejercicio metodológico que sugiere García es retomar la pregunta de investigación formulada como base para el desarrollo del proyecto, ontología de la investigación científica, con el objetivo de instrumentalizarla en la construcción de la metodología y asumirla desde una perspectiva teleológica (Ríos y Mesa, 2009). A partir de este momento se inicia la caracterización y construcción del sistema desde sus componentes: los límites, los elementos, las estructuras, los procesos y los niveles de análisis.

Estos componentes del sistema complejo se definen en el desarrollo de la investigación desde la observación, la cual siempre está ligada a preconcepciones teóricas del investigador. De este modo, el marco epistemológico se establece en el sistema complejo por el tipo de pregunta o el conjunto de estas que orienta la pesquisa. A continuación se describen brevemente los componentes de un sistema, de acuerdo con García (2006):

a. Los límites del sistema: los sistemas complejos carecen de límites tanto físicos como en su problemática. El autor propone establecer "límites más o menos arbitrarios” (p. 48) del sistema que se va a estudiar. Estos límites incluyen además el conjunto de conceptos que el investigador ha construido teóricamente, el tipo de fenómeno y las escalas temporales y espaciales. Esto define lo que queda dentro y fuera del sistema. Lo que está por fuera, debe ser también analizado y es lo que el autor considera como "condiciones de contorno o condiciones de los límites" (p. 49). A su vez, la escala temporal indica cuál ha sido la velocidad de cambio del fenómeno; por tanto, la introducción de un determinante histórico es fundamental, en ocasiones, para comprender las reorganizaciones del sistema. 
b. Los elementos: son “interdefinibles", es decir, se determinan de forma mutua. Se organizan en unidades que también son complejas o subsistemas. Estas interactúan entre sí y a partir de estas relaciones se determina la estructura del sistema.

c. La estructura: está dada por el conjunto de relaciones que se establecen entre los subsistemas y no por los elementos.

d. Los niveles de procesos y los niveles de análisis: el análisis de la dinámica del sistema tiene los procesos como foco principal. El autor diferencia tres niveles: el primero, incluye aquellos procesos que afectan directa y localmente el proceso central; el segundo, se refiere a los procesos a escala regional o nacional que tienen efecto sobre los procesos de primer nivel; y tercero, procesos de carácter internacional o global con efectos sobre los procesos de segundo nivel y de primer nivel.

El sistema establecido a partir de la definición de sus componentes sirve como insumo para determinar los observables y los datos que se deberán obtener de estos durante el proceso de investigación. De este modo, se definen los métodos y los instrumentos necesarios para la recolección de la información necesaria con el fin de dar respuesta a la pregunta de investigación inicial.

\section{La agroecología como transdisciplina}

La denominación de la agroecología como una interdisciplina o transdisciplina es una discusión recurrente en el ámbito agroecológico. Con la introducción de variables sociales se ha planteado la evolución de la agroecología como una ciencia interdisciplinaria (Toledo, 20I2), que identifica las bases ecológicas y ambientales de un desarrollo socioeconómico más sostenible (Guzmán-Casado, González y Sevilla, 1999).

Para establecer si la agroecología se emplaza en una interdisciplina, es necesario hacer un análisis de las definiciones de este término, que históricamente ha tenido múltiples acepciones. Palmade (1979, p. 22), la define así:

"Interacción existente entre dos o más disciplinas. [No obstante, esta] puede ir desde la simple comunicación de ideas, hasta la integración mutua de los conceptos directivos, de la epistemología, de la terminología, de la metodología, de los procedimientos, de los datos y de una organización de la investigación y la enseñanza correspondiente". 
Ander-Egg (1994, p. 28) establece que la "problemática de la interdisciplinariedad evoca la idea de puesta en común y de intercambio entre diferentes disciplinas. Es una forma de preocupación por tender hacia la unidad del saber, habida cuenta de la complejidad de lo real”. Aquí, lo más importante para el autor es el concepto de interacción y entrecruzamiento de ideas y conceptos de las disciplinas.

Esta perspectiva puede ser respaldada por el aporte de Jean Piaget, quien argumenta que la interdisciplinariedad se da "donde la cooperación entre varias disciplinas o sectores heterogéneos de una misma ciencia llevan a interacciones reales, es decir, hacia una cierta reciprocidad de intercambios que da como resultado un enriquecimiento mutuo" (1979, p. 67). Esto explica la orientación metodológica que las investigaciones en agroecología, ya sea desde aproximaciones cuantitativas o cualitativas, lejanas aún de un proceso de integración de carácter complejo.

En contraste, la transdisciplinariedad es un término "inventado para expresar la necesidad de una feliz transgresión de las fronteras entre las disciplinas, de una superación de la pluri y de la interdisciplinariedad” (Nicolescu, 1998, p. 3). Además para Newell y Zarri (en McDonnell, 1998, p. 3) esta implica una acción integradora y transformadora del conocimiento, la cual es necesaria para comprender y tratar la complejidad de los fenómenos agroecológicos: "La transdisciplinariedad es la transformación e integración del conocimiento desde todas las perspectivas interesadas para definir y abordar problemas complejos”. Además, implica un proceso de reflexión de la acción epistemológica del sujeto sobre la realidad y del conocimiento mismo; por esta razón Klein establece que el proceso transdisciplinario consiste en "practicar el conocimiento en un modo reflexivo, que reconoce y no niega la pluralidad inherente y la complejidad de la condición humana" (en McDonnell, 1998, p. 4).

La transdisciplinariedad de Nicolescu, parte de reconocer la existencia de niveles de realidad diferentes entre sí, tanto en su estructura como en la forma de relación. Además, plantea:

"La transdisciplinariedad es complementaria al enfoque disciplinario: hace emerger de la confrontación de las disciplinas nuevos datos que las articulan entre sí, y nos ofrece una visión de la naturaleza y de la realidad. No busca el dominio de muchas disciplinas, sino la apertura de todas las disciplinas a aquellos que las atraviesan y las trascienden” (1998, p. 89). 
A su vez, la Carta de la Transdisciplinariedad (Convenio de Arrábida, 1994), propone tres características fundamentales de la actitud y visión transdisciplinaria: el rigor en los argumentos en el análisis de la realidad; la apertura a la aceptación de lo desconocido, lo inesperado y lo imprevisible; y la tolerancia que implica el reconocimiento de ideas y verdades opuestas a las propias.

Estos argumentos soportan la idea de proponer la agroecología como una transdisciplina con un objeto de estudio particular, el cual sugiere un nuevo enfoque epistemológico y metodológico que pretende superar la fragmentación de la ciencia clásica. Para comprender la realidad interactuante y totalizadora es mandatorio valorar fenómenos interconectados en forma recíproca.

La agroecología es una disciplina nueva que se sustenta en los conocimientos, las teorías y las metodologías de otras disciplinas que aportaron en la construcción de su ya mencionado nuevo objeto de estudio, que le es propio e intransferible. Este es un factor que la diferencia de la ecología, la agronomía, la antropología, la economía y otras áreas que la soportan. A su vez, el enfoque de sistemas complejos considera dos dimensiones: la temporal y la espacial. Dimensiones que se pueden comprender desde una base transdisciplinaria, donde la aproximación al objeto de estudio puede hacerse desde múltiples metodologías.

\section{Conclusiones}

El desarrollo de la agroecología requiere como condición sine qua non la definición de su objeto de estudio por parte de los agroecólogos. Esta reflexión epistemológica se desarrolla a medida que la agroecología continúa en un proceso de consolidación como transdisciplina científica. Sin embargo, una pregunta que surge al analizar los asuntos epistemológicos de la agroecología es épor qué los planteamientos de los discursos políticamente correctos que la presentan como una ciencia inter o transdisciplinar y holística no corresponden con la aplicación operativa y funcional en las investigaciones agroecológicas? Estas aún conservan una línea evidentemente cartesiana y parcializada, en una esfera disciplinar particular que, si bien es válida, se aleja tanto del objeto de estudio propuesto, como de los discursos que la agroecología promulga.

$\mathrm{Al}$ instaurar a la agroecología como una transdisciplina, supone la existencia de niveles de complejidad y de relación, lo que posibilita abordar la complejidad de la realidad desde diversos enfoques epistemológicos. Esto puede aportar a la construcción del conocimiento de las dinámicas socioecológicas de los 
agroecosistemas, lo que permite entender su resiliencia. Por consiguiente, el enfoque epistemológico elegido por un investigador debe guiar de manera coherente la elección del enfoque metodológico. En particular, la agroecología invita a aproximarse a la realidad desde el enfoque de sistemas complejos.

El enfoque sistémico como marco epistemológico de la investigación en agroecología no pretende predecir eventos futuros o establecer relaciones causaefecto, tal como se ha sugerido históricamente en la ciencia convencional. En su lugar, tiene como propósito la comprensión de los fenómenos en un lugar y tiempo, y por tanto estos fenómenos no son susceptibles de ser generalizados. Finalmente, se resalta que el presente artículo es un aporte en la definición el objeto de estudio de la agroecología, como la resiliencia socioecológica que deberá ser analizada en los agroecosistemas, los cuales se constituyen en el objeto de conocimiento. De igual forma, este trabajo es un aporte a la formación de investigadores en agroecología, proceso que requiere de bases sólidas tanto desde lo filosófico como desde lo pedagógico, y al mismo tiempo la necesidad de espacios de discusión que permitan la consolidación de la agroecología como ciencia transdisciplinar.

\section{Referencias}

Abbona, E., Sarandón, S., Marasas, M. y Astier, M. (2007). Ecological Sustainability Evaluation of Traditional Management in Different Vineyard Systems in Berisso, Argentina. Agriculture, Ecosystems E- Environment, II9(3), 335-345. Altieri, M. (1995) Agroecology: the Science of Sustainable Agriculture. Boulder: Westview Press.

Altieri, M. (1999). Agroecología: bases científicas para una agricultura sustentable. Montevideo: Nordan-Comunidad.

Altieri, M., Lana, M., Von Hertwig, H., Venturi, M., Dos Santos, A., Comin, J. y Lovato, P. (2012). Aumento do rendimiento dos cultivos através da supressão de plantas espontâneas em sistemas de plantio direto orgânico em Santa Catarina, Brasil. Agroecología, 7(I), 63-7I.

Ander-Egg, E. (1994). Interdisciplinariedad en educación. Buenos Aires: Editorial Magisterio del Río de la Plata.

Arnold, M. y Osorio, F. (1998). Introducción a los conceptos básicos de la Teoría

General de Sistemas. Cinta de Moebio, (3). Recuperado el 29 de septiembre de 2013, de http://www.redalyc.org/articulo.oa?id=I0100306 
Barrera-Bassols, N. y Toledo, V. (2005). Ethnoecology of the Yucatec Maya: Symbolism, Knowledge and Management of Natural Resources. Journal of Latin American Geography, 4(I), 9-4I.

Caporal, F., Costabeber, J. y Paulus, G. (2009). Agroecologia: uma ciência do campo da complexidade. Brasília. Recuperado el I7 de junio de 20I4, de http://www.emater.tche.br/site/arquivos_pdf/teses/ Agroecologiaumacienciadocampodacomplexidade.pdf

Convenio de Arrábida (I994). Comité de redacción: Lima Freitas, Edgar Morin y Basarab Nicolescu. Recuperado el is de octubre de 20I3, http://www.filosofia. org/cod/cig94tra.htm

Conway, G. (1987). The Properties of Agroecosystems. Agricultural Systems, 24(2), 95-II7.

Gallopin, G. (200I). Science and Technology, Sustainability and Sustainable Development. LC/R.208I. ECLAC. Recuperado el I7 de junio de 20I4, de http://repositorio. cepal.org/bitstream/handle/II362/31809/So2157.pdf?sequence=I

García, R. (2006). Sistemas complejos. Conceptos, método y fundamentación epistemológica de la investigación interdisciplinaria. Barcelona: Gedisa.

Gliessman, S. (2002). Agroecología: procesos ecológicos en agricultura sostenible. Turrialba: Centro Agronómico Tropical de Investigación y Desarrollo.

Gómez, L., Ríos-Osorio, L. y Eschenhagen, M. (2013). Agroecology Publications and Coloniality of Knowledge. Agronomy for Sustainable Development, 33(2), 355-362.

Guzmán-Casado, G., González, M. y Sevilla, E. (1999). Introducción a la agroecología como desarrollo rural sostenible. Madrid: Ediciones Mundiprensa.

Hecht, S. (1999). La evolución del pensamiento agroecológico. En M. Altieri (Ed.), Agroecología: bases científicas para una agricultura sustentable (pp.15-30). Montevideo: Nordan-Comunidad.

Holling, C. (1973). Resilience and Stability of Ecological Systems. Annual Review of Ecology and Systematics, (4), I-23.

Holt-Giménez, E. y Patel, R. (2010). Rebeliones alimentarias. Crisis y bambre de justicia. Mataró: El Viejo Topo.

Hoobbelink, H. (1987). ¿Más allá de la revolución verde: las nuevas tecnologías genéticas para la agricultura, desafío o desastre? Barcelona: Lerna.

Junge, X., Jacot, K., Bosshard, A. y Lindemann-Matthies, P. (2009). Swiss People’s Attitudes towards Field Margins for Biodiversity Conservation. Journal for Nature Conservation, ${ }_{17}(3)$, I50-159. 
León, T. (2012). Agroecología: la ciencia de los agroecosistemas. La perspectiva ambiental. Bogotá D.C.: Instituto de Estudios Ambientales/Universidad Nacional de Colombia.

Malpartida, A. y Lavanderos, L. (1995). Aproximación a la Unidad SociedadNaturaleza, el ecotomo. Revista Chilena de Historia Natural, 68, 419-427.

Martínez-Negrete, M., Martínez, R., Joaquín, R., Sheinbaum, C., y Masera, O. (2013). Is Modernization Making Villages more Energy Efficient? A Long-Term Comparative End-Use Analysis for Cheranatzicurin Village. Energy for Sustainable Development, I7 (5), 463-470.

McDonnell, G. (mayo, I998). ¿Qué es la transdisciplinariedad? Documento presentado en el Simposio Internacional de Transdisciplinariedad. Unesco.

Méndez, V. y Gliessman, S. (2002). Un enfoque interdisciplinario para la investigación en agroecología y desarrollo rural en el trópico latinoamericano. Manejo integrado de plagas y agroecología, (64), 5-16.

Mora-Delgado, J. (2008). Persistencia, conocimiento local y estrategias de vida en sociedades campesinas. Revista de Estudios Sociales, (29), 3-17.

Morin, E. (1999). Los siete saberes necesarios para la educación del futuro. París: Unesco. Morin, E. (2003). Introducción al pensamiento complejo. Barcelona: Gedisa.

Norgaard, R. (1997). Metodología y práctica de la agroecología. En M. Altieri (Ed.), Agroecología. Bases científicas para una agricultura sustentable (pp. I5-28). Montevideo: Nordan Comunidad.

Nicolescu, B. (1998) La transdisciplinariedad, una nueva visión del mundo. Manifesto. París: Du Rocher.

Palmade, G. (1979). Interdisciplinariedad e ideologías. Madrid: Narcea ediciones. Piaget, J. (1979). L'interdisciplinarité: problèmes d'enseignement et de recherche dans les universités. París: OCDE.

Reijntjes, C., Haverkort, B. y Waters-Bayer, A. (1992). Farming for the Future: an Introduction to Low-External Input and Sustainable Agriculture. Londres: Mac Millan.

Ríos, L., Ortiz, M. y Álvarez Del Castillo, X. (2009). An Epistemology for Sustainability Science: a Proposal for the Study of the Health/Disease Phenomenon. International Journal of Sustainable Development E. World Ecology, I6(I), $48-60$.

Ríos, L. y Mesa, A. (2009). Introducción al pensamiento científico en microbiología. Medellín: Fondo Editorial CIB. 
Rosset, P. y Martínez-Torres, M. (2012). Rural Social Movements and Agroecology: Context, Theory and Process. Ecology and Society, $17(3), 17-29$.

Salas-Zapata, W., Ríos-Osorio, L. y Álvarez-Del Castillo, J. (20II). La ciencia emergente de la sustentabilidad. Interciencia, 36(9), 699-706.

Sevilla, E. (20II). Sobre los orígenes de la agroecología en el pensamiento marxista y libertario. La Paz: Agruco, Plural Editores, CDE y NCCR.

Snow, C. (2000). Las dos culturas. Madrid: Ediciones Nueva Visión.

Toledo, V. (2012). La agroecología en Latinoamérica: tres evoluciones, una misma transformación. Agroecología, 6, 37-46.

Ulloa, A. (200I). El nativo ecológico: movimientos indígenas y medio ambiente en Colombia. Movimientos sociales, Estado y democracia en Colombia. Bogotá D.C.: Icanhces/ Universidad Nacional de Colombia.

Vargas, G. (2003). Tratado de epistemología: fenomenología de la ciencia, la tecnología y la investigación social. Bogotá D. C.: San Pablo.

Walker, B., Holling, C., Carpenter, S. y Kinzig, A. (2004). Resilience, Adaptability and Transformability in Social-Ecological Systems. Ecology and Society, 9(2), 5-10.

Wezel, A., Bellon, S., Doré, T., Francis, C., Vallod, D. y Davis, C. (2009). Agroecology as a Science, a Movement and a Practice. A Review. Agronomy for Sustainable Development, 29(4), 503-515.

Zerda, A. (2003). Derechos de propiedad intelectual del conocimiento vernáculo. Análisis y propuesta desde la economía institucionalista. Bogotá D.C.: Universidad Nacional de Colombia/Facultad de Educación, Antropos LTDA. 